\title{
IMPORTANCIA DE LAS FINANZAS PERSONALES EN LA SALUD FINANCIERA: UNA REFLEXIÓN TEÓRICA
}

\author{
Alan Enrique Tejeda-Hernández \\ Universidad Cristóbal Colón. Boca del Río, Veracruz, México. \\ alante1492@gmail.com \\ Arturo García-Santillán \\ Universidad Cristóbal Colón. Boca del Río, Veracruz, México. \\ Michelle Valerie Martínez-Rodríguez \\ Universidad Cristóbal Colón. Boca del Río, Veracruz, México.
}

Recepción Artículo: 13 enero 2021

Admisión Evaluación: 13 enero 2021

Informe Evaluador 1: 17 enero 2021

Informe Evaluador 2: 18 enero 2021

Aprobación Publicación: 19 enero 2021

\section{RESUMEN}

Lograr llevar a cabo finanzas personales suele parecer una tarea muy sencilla, sin embargo, para lograrlo es necesario una serie de conocimientos y habilidades financieras. No obstante, las personas no cuentan con una amplia alfabetización financiera, lo cual hace difícil esta labor de alcanzar una salud financiera, misma que se puede lograr a través de la educación financiera, pues la falta de esta misma afecta en los niveles de ingreso de las personas y como consecuencia no recurren al ahorro, no planifican un presupuesto, presentan sobreendeudamiento, entre otros. A su vez, al no estar incluidos financieramente no les permite aprovechar las oportunidades que el Sistema Financiero brinda como por ejemplo: el acceso al ahorro o a la inversión. Lo mencionado anteriormente trae como consecuencia que los individuos difícilmente puedan alcanzar un nivel de bienestar financiero. Por ello, el propósito de este ensayo es destacar la importancia que tiene la educación financiera en la vida de las personas, con el fin de administrar sus finanzas personales, lo que ayudaría a enfrentar contingencias económicas que se presentan con frecuencia.

Palabras clave: educación financiera; finanzas personales; inclusión financiera; bienestar financiero

\section{ABSTRACT}

Importance of personal finance in financial health: A theoretical reflection. Achieving a personal finance could sound a very simple task, however, it's necessary to have some financial knowledge and financial skills. Nevertheless, people don't seem to have high financial literacy levels, this 


\section{IMPORTANCIA DE LAS FINANZAS PERSONALES EN LA SALUD FINANCIERA: UNA REFLEXIÓN TEÓRICA}

makes it difficult to achieve financial health, which can be achieved through financial education, so the lack of this impacts on income levels of people and as a result they don't make savings, do not plan a budget, they get over-indebtedness, among others. Furthermore, being not included financially does not allow people to take the opportunities that the Financial System offers, such as: access to savings or investments. The aforementioned brings as a consequence that individuals can hardly reach a level of financial well-being. Therefore, the purpose of this essay is to highlight the importance of financial education in people's lives, in order to manage their personal finances, which would help to face frequently encountered economic contingencies.

Keywords: financial literacy; personal finance; financial inclusion; financial well-being

\section{INTRODUCCIÓN}

Muchas de las personas quisieran tener una mejora en sus finanzas personales a través de controlar mejor sus gastos, ahorrar para lograr una meta o incluso el salir de una deuda. La educación financiera puede ser el arma para lograr acercarse a estos objetivos (Banco Mundial, 2014).

En la misma idea Joo y Garman (1998) refieren que el ritmo de sobreendeudamiento de las personas aumenta más rápido que la misma inflación y lo pudo observar en datos obtenidos donde cada año el número de personas en crisis financiera en los EE. UU., donde lejos de disminuir, aumentaba.

Dentro de los hallazgos en la investigación de Brown, Mount, Lamborn y Steinberg (1993) identificaron que los colaboradores sufrían estrés debido a problemas relacionados con el dinero. Asimismo, algunas de las causas se relacionaban con el uso imprudente del crédito, sobreendeudamiento, malas decisiones del gasto, mal manejo del dinero y presupuesto ineficiente al llegar a fin de mes.

Como resultado de los anteriores problemas de los empleados, estos autores señalan que muchas empresas en los Estados Unidos han optado por equipar a sus colaboradores con conocimientos financieros y habilidades que aporta la educación financiera para que logren un buen manejo de sus finanzas personales.

Una escasa gestión financiera personal afecta la productividad en el lugar de trabajo, tal como Io refieren Garman, Leech y Glable (1996), también hallaron que diversos colaboradores en los EE. UU., Ilegaban a tal grado de estrés por sus malos hábitos financieros y en consecuencia esto repercutía negativamente en su productividad laboral.

Con el fin de obtener el éxito financiero se deben lograr cinco objetivos financieros en la vida, según Garman y Forgue (1997):

Obtener el máximo de ganancias y riqueza.

Practicar un consumo eficiente.

Encontrar satisfacción con la vida.

Alcanzar seguridad financiera.

Acumular tal riqueza para la jubilación y así tener un patrimonio que dejar.

Lo anterior parece muy sencillo, sin embargo, en la práctica es un tema tan complejo que demanda numerosos conocimientos y habilidades financieras. Una de las principales razones por la cual los individuos suelen presentar diversos problemas financieros es el analfabetismo financiero.

De acuerdo con la investigación llevada a cabo por Garman y Forgue (1997) la población americana no se encuentra ampliamente desarrollada en el campo de educación financiera.

Estos autores analizaron la falta de conocimiento en finanzas personales, las complejidades que tiene una vida financiera, las tantas opciones que se tienen para poder lograr una decisión financiera asertiva, así como la falta de tiempo entre los individuos para aprender sobre finanzas personales, mismas que van estrechamente relacionadas con una buena educación financiera. 
Tal como menciona Joo (1998), el estrés financiero y la situación financiera de una persona se relaciona directamente sobre qué tan satisfecho financieramente está o no y sobre cómo se encuentra su vida financiera. Por lo tanto, los colaboradores con un alto nivel de bienestar financiero presentaron características buenas en cuanto a mejores niveles de rendimiento, un menor grado de ausentismo y menor tiempo de trabajo utilizado para atender sus asuntos financieros.

Asimismo, el nivel de inclusión financiera garantiza que los individuos puedan familiarizarse con términos de finanzas y como resultante la utilización a un grado meramente positivo de productos que le resulten benéficos, es por eso por lo que ha despertado el interés para diversas instancias tanto del sector privado como el público, incluyendo los agentes del mercado, por lo que señalan, es tarea de todos proporcionar cimientos de educación financiera (Banco Mundial, 2018).

Y, ¿qué es la educación financiera?, según Lusardi y Mitchell (2007), es un proceso en el cual una persona (consumidor o inversionista) logra comprender sobre los conceptos, productos financieros e inversiones a través de la información, educación y el asesoramiento, para así lograr tener conocimiento de los riesgos y oportunidades financieras.

En distintos países se ha encontrado que el poco grado de educación financiera influye negativamente en el bienestar del hogar, por ejemplo, los individuos menos alfabetizados financieramente escogen préstamos con tasas altas de interés, tienen una participación menor en los mercados financieros o sistema bancario, y no planifican correctamente para su jubilación (Kumar, Barua, Jacob \& Varma, 2012).

Es por eso que desde 2010, cerca de 55 países se han comprometido a sumar esfuerzos sobre este tema, y más de 30 han implementado o bien, se encuentran planeando una estrategia nacional (Banco Mundial, 2018).

Existen diversas causas que originan problemas financieros en la sociedad y es que el entorno financiero se ha vuelto más complejo y retador tanto para instituciones y negocios, a la par de los hogares e individuos. A su vez, el incremento del costo de vivir y de los cambios en los precios de bienes y servicios, así como también el incremento de la necesidad de querer más de los mismos.

Aunado a lo anterior, se incrementan las necesidades de las personas, pero muchas veces los ingresos no aumentan al mismo tiempo, esto da como resultado ser un blanco fácil para solicitar un crédito 0 un préstamo, lo cual, si no se cuenta con conocimientos financieros necesarios se pone en peligro el buen manejo de las finanzas de una persona (Zakaria, Jaafar \& Marican, 2012).

Estos mismos autores indican que el sobreendeudamiento ha tenido un incremento en las últimas décadas pues las personas intentan tener un mejor nivel de vida y complacer sus deseos acudiendo a un consumismo desmesurado tanto en bienes como en servicios.

En consecuencia, para lograr obtener un bienestar financiero se requiere de una alfabetización financiera a través de la cual se adquieran habilidades, actitudes y comportamientos que nos encaminen a obtenerlo (OCDE, 2013).

Por eso mismo recae la importancia de una buena educación financiera, pues autores como Billimoria, Penner y Koonte (2013) la definen como la provisión de servicios educativos como instruir 0 incluso materiales elaborados de acuerdo a las necesidades que se requieran para que los individuos puedan aumentar sus conocimientos financieros y habilidades y todo este conjunto de conocimientos no tendría sentido sobre todo si no se aplican en el mundo financiero que hoy en día son muchos los servicios y productos que se ofrecen.

El Banco Mundial (2014) reveló cifras sobre las oportunidades que hay en el manejo de las finanzas personales en estudios realizados entre Colombia y México donde:

Los individuos no respetan sus planes financieros a pesar de elaborarlos. Más del $70 \%$ dijo haber presupuestado, sin embargo, un $25 \%$ no sabía cuánto había gastado la semana anterior. 


\section{IMPORTANCIA DE LAS FINANZAS PERSONALES EN LA SALUD FINANCIERA: UNA REFLEXIÓN TEÓRICA}

La planificación financiera suele ser a corto plazo y el ahorro es limitado. En México se encontró que solo el $34 \%$ de los encuestados puede cubrir gastos inesperados mientras que el $43 \%$ planifica a una semana o menos. Por otro lado, se reveló que sólo el $28 \%$ de adultos menores de 60 años puede cubrir los gastos de retiro.

No todos se les enseña a manejar su dinero. En la mayoría de los mexicanos y colombianos revelaron que la única información que recibieron sobre la gestión de su dinero es a través de sus padres como por cada $\$ 100$ pesos que recibas, ahorras $\$ 10$, o como poner el monto del gasto al cual será destinado cada uno.

Oton y Torrento (2017) hacen referencia a la falta de conocimiento financiero o a su vez la falta de comprensión sobre cómo se debe administrar, ahorrar, o bien, invertir dinero.

Respecto al ahorro, según la CONDUSEF (2018) refiere el mejor momento para empezar a hacerlo y lograr nuestras metas o alcanzar una vejez digna es en el presente, sin embargo, un gran porcentaje no lo hace ya que según datos de la Encuesta Nacional de Inclusión Financiera de 2012 afirma que sólo un $40 \%$ de la población mexicana no ahorra y vive con la filosofía "Dios proveerá", sin embargo, también la población debe tomar en cuenta la frase "ayúdate que yo te ayudaré", y de este modo no dejar al azar el destino financiero.

También, esta institución menciona que actualmente alrededor de la mitad de los mexicanos sigue creyendo que no es necesario ahorrar para la vejez y piensan que sus hijos deben ser quienes se ocupen de ellos mismos en el futuro, sin embargo, nada garantiza que suceda eso, pues el futuro es incierto

Actualmente, el existente bajo nivel de educación financiera se ha relacionado con el abuso del crédito, así como el llevar una vida que va más allá de lo que les permitiría vivir a los individuos. Es por eso por lo que el manejo de las finanzas personales ayuda a mejorar el entorno financiero de las personas ya sea en el hogar u otros aspectos personales de la vida que tienen que ver con la administración del dinero donde se incluyen herramientas como presupuestos, ahorro e inversiones, todo esto para ayudar a alcanzar las metas personales (Munohsamy, 2005).

Por otro lado, estudios sociológicos proponen cuatro preceptos que indican que las personas están presentando felicidad y bienestar, los cuales son: situación laboral que presentan, situación familiar en la que se encuentran, el estado de salud que tienen y la situación económica en la que viven. Por lo tanto, cuando las personas se encuentran estables en las áreas anteriormente mencionadas son felices y presentan bienestar (0’Neill, Sorhaindo, Xiao \& Garman, 2005).

\section{DESARROLLO}

\section{La Inclusión Financiera}

La Comisión Nacional Bancaria y de Valores (CNBV, 2009) refiere a la inclusión financiera como el acceso y uso de un portafolio de productos y servicios financieros que llega a la mayor parte de la población adulta con información clara y concisa para satisfacer la demanda en aumento, bajo un marco regulatorio apropiado.

Por otro lado, la Red Internacional para la Educación Financiera (INFE por sus siglas en inglés) ha definido la inclusión financiera como el proceso de promoción de un acceso asequible, oportuno y adecuado a una amplia gama de productos y servicios financieros regulados, ampliando su uso a todos segmentos de la sociedad a través de la implementación de enfoques existentes e innovadores a la medida, incluye la sensibilización y educación para promover el bienestar financiero, así como la inclusión económica y social (OCDE, 2013).

Asimismo, la Fundación de Estudios Financieros FUNDEF (2017) presentó un mapa mundial en donde se puede observar el porcentaje de inclusión financiera que hay en distintos países alrededor 
del mundo según un estudio llevado a cabo en 148 países por donde midieron variables como ahorro, préstamos, pagos y la forma de administrar sus riesgos. El estudio arrojó el siguiente mapa:

Tabla 1.

Adultos con una cuenta en una institución financiera formal. Fuente: FUNDEF (2017)

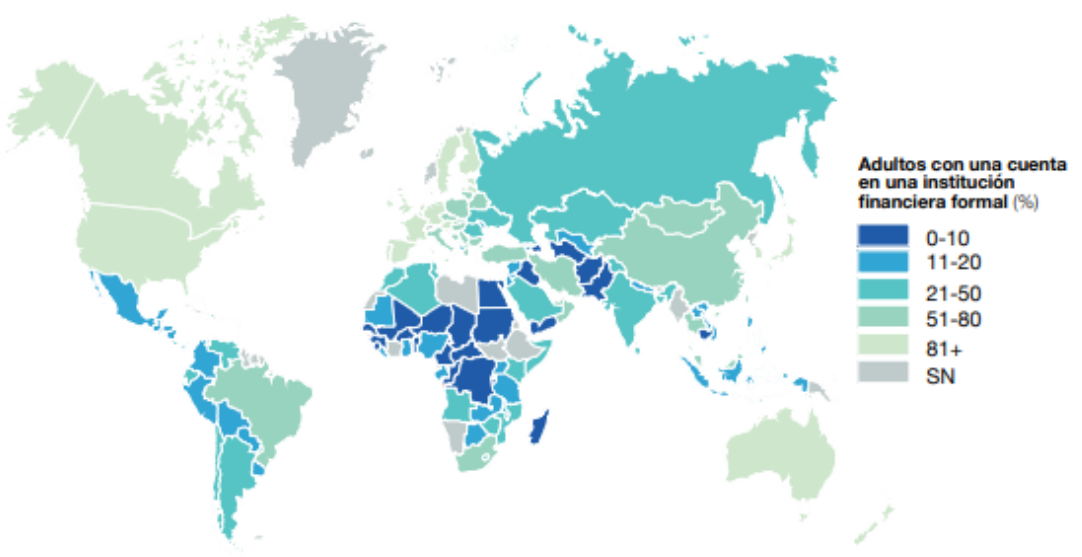

En el mapa anterior se puede apreciar el porcentaje de adultos con una cuenta en una institución financiera formal que existe en cada país. Las zonas en las que se puede apreciar un alto son en países como Estados Unidos, Australia o China. Por otro lado, en el continente africano se puede observar que muy pocos individuos tienen una cuenta formal.

Mientras en el contexto mexicano se ubica en un rango de entre $11-20 \%$, lo cual, se puede inferir que es un área de oportunidad para este país en fomentar cada vez más la inclusión financiera y que la gente pueda aprovechar los servicios que se ofrecen como el de una cuenta de ahorro formal bajo el esquema regulado por el sistema financiero.

\section{Educación Financiera}

El término educación financiera ha ido evolucionando pues antes se le solía llamar para hacer referencia como administración del dinero o conocimiento financiero donde se utilizaban para medir diversas variables como seguros, préstamos, crédito, presupuesto, entre otros (Danes \& Hira, 1987).

Billimoria, Penner y Koonte (2013) refieren que la educación financiera quiere decir que se deben de proveer de servicios educativos como instruir o incluso materiales elaborados de acuerdo a las necesidades que se requieran para que los individuos puedan aumentar sus conocimientos financieros y habilidades en este caso el de los jóvenes y todo este conjunto de conocimiento no tendría sentido sobre todo si no se aplican en el mundo financiero que hoy en día son muchos los servicios y productos que se ofrecen.

Para la OCDE (2005) es definida como aquel proceso por el que los consumidores ya sea financieros 0 inversores optimizan su comprensión de los productos financieros, conceptos y riesgos a través de la información, la enseñanza y/o el asesoramiento objetivo, por lo que buscan el desarrollo de las habilidades y la confianza con la finalidad de adquirir mayor conciencia del riesgo y opor- 


\section{IMPORTANCIA DE LAS FINANZAS PERSONALES EN LA SALUD FINANCIERA: UNA REFLEXIÓN TEÓRICA}

tunidad financiera, para tomar decisiones informadas, saber dónde acudir para pedir ayuda y tomar cualquier acción eficaz para mejorar su bienestar financiero.

Aunado a lo anterior, la educación financiera debe cubrir las opciones de inversión y transmitir la comprensión correcta de los conceptos relacionados con la inflación, los intereses y los impuestos (Lusardi, 2009).

Por esta razón, el conocimiento financiero se aplica para proporcionar bienestar financiero a las personas y para eso es necesario una combinación de habilidades, actitudes y comportamientos (OCDE, 2013).

Un dato importante para destacar es que el nivel alto de escolaridad no garantiza un alto nivel de educación financiera. Lo anterior, lo confirman estudios de Lusardi y Mitchell, (2011), al buscar una correlación entre ambas, se concluyó que incluso aún con un grado elevado de escolaridad, el nivel de educación financiera tendió a ser bajo, por lo tanto, este no puede ser un indicador del nivel de educación financiera.

Según la OCDE, el nivel de educación financiera de una persona puede medirse con base en sus conocimientos en materia financiera como lo pueden ser la realización de presupuestos, la administración del dinero, la planificación a corto y largo plazo y a través de escoger productos financieros que brinden un mayor beneficio (Atkinson \& Messy, 2012).

\section{Manejo de las Finanzas Personales}

Garman et al. (1996) manifiesta que el grado de estrés está relacionado con malos hábitos financieros, lo que puede repercutir en varias áreas de la vida de una persona, por ejemplo, afectar en su productividad laboral.

Las finanzas personales se definen como el proceso que tiene un individuo de controlar sus ingresos y organizar sus gastos a través de una planificación financiera detallada. Aprender a hacer un seguimiento del dinero que ingresa y adaptar este dinero como un traje a la medida que se ajuste a los gastos proporciona una forma sistemática y eficiente de manejar los ingresos de un individuo (Munohsamy, 2005)

Para Huston (2010) la educación financiera se basa en dos niveles, el primero es el conocimiento y el segundo su uso, por lo que el modelo de educación financiera que propone es que la educación financiera, el conocimiento y aplicación del individuo, en materia financiera personal, se puede obtener como resultado la buena toma de decisiones financieras, con lo que a su vez brindará un bienestar financiero, mismo que se ampliará más adelante.

Borghino (2012) hace mención sobre algunos puntos relevantes para llevar a cabo un buen manejo de finanzas personales las cuales son: mantener una educación financiera, estar pendiente del crédito, pago de deudas en fecha correspondiente y ahorro constante.

En el estudio de Oton y Torrento (2017), reveló que las personas que presentaban un nivel justo de bienestar financiero obtuvieron un nivel moderado de educación financiera, un satisfactorio nivel de comportamiento financiero y un bajo nivel de estrés financiero. Los colaboradores estudiados eran exactamente iguales en su estado de bienestar financiero independientemente de su edad, sexo, estado familiar, permanencia y empleo.

\section{Bienestar Financiero o Salud Financiera}

Para Joo (1998) el bienestar financiero refiere a que este término no es más que un nivel de salud financiera, la cual, incluye satisfacción con aspectos materiales y no materiales de la situación financiera, percepción de la estabilidad financiera, incluyendo suficiencia de recursos financieros, materiales e inmateriales que cada individuo posee.

En la misma idea, Kim (2000) refiere que el término de bienestar financiero es básicamente un 
resultado que se da al llevar a cabo un proceso de administración financiera que incluyen las actitudes financieras, el conocimiento financiero y los comportamientos que brinda la educación financiera, pues se encontró que aquellos que tuvieron bases de educación financiera gozaban de mejor salud, menos estrés financiero y una mejor satisfacción financiera.

Por otro lado, León (2006) define el término como el estado financiero en el que se encuentra la persona, en otras palabras, se refiere al grado en que las personas sienten certidumbre económica o confianza con su dinero.

Kim (2000) realizó una investigación en una empresa de seguros de Estados Unidos donde observó variables como actitudes, comportamientos, conocimientos, los efectos que obtenían a través de la educación financiera y como influía en su bienestar financiero.

De los resultados que destacaron en este estudio fue que, entre menor salud financiera, la productividad fue más baja, mientras que, aquellos colaboradores que presentaban mayor grado de salud financiera mostraron un índice mayor de productividad, menor ausentismo y menor pérdida de tiempo en asuntos relacionados al dinero.

Asimismo, el grado de bienestar financiero se hizo notar a través de una mayor productividad laboral, pues los colaboradores presentaron actitudes positivas y un mayor conocimiento en el ámbito de las finanzas personales.

Billimoria et al. (2013) agregan que a través de la educación financiera los individuos pueden aumentar sus habilidades en materia financiera.

Además, el Center for Financial Services Innovation (2016) menciona ocho indicadores en las que un individuo puede saber si cuenta con una buena salud financiera. Estos indicadores tienen que ver con gastar menos de lo que se gana, pagar facturas en tiempo y en su totalidad, tener suficientes ahorros en productos financieros líquidos, poseer suficientes ahorros 0 activos a largo plazo, tener un nivel de deuda sostenible, contar con un historial crediticio bueno, tener segur os apropiados debido a que no siempre se tiene el seguro de acuerdo a las necesidades de la persona y por último, un plan de gastos para el futuro.

\section{Habilidad financiera.}

En 2008, el presidente del Consejo Consultivo de Educación Financiera de los EE.UU., dijo que, si bien la crisis tiene muchas causas, es innegable que el analfabetismo financiero es una de las causas principales causas. Lamentablemente, demasiados estadounidenses no tienen las habilidades financieras básicas necesarias para desarrollar y mantener un presupuesto para entender el crédito, la inversión o para aprovechar incluso el sistema bancario (Lusardi, 2011).

Huston (2010) afirma que una persona que posee educación financiera debe contar con habilidades necesarias que le permitirán tomar decisiones acertadas en el mercado financiero.

El término de habilidad financiera se refiere a la facultad que tiene una persona para que a partir de los conocimientos financieros que tiene pueda llegar a tomar una decisión (Zait \& Bertea, 2015).

Chinen y Endo (2012) refieren que los individuos que cuenten con habilidades financieras además de poder tomar mejores decisiones financieras, sus problemas económicos se verán disminuidos al no tener sobreendeudamiento, planificará para su futuro, pagará sus tarjetas de crédito, priorizará sus gastos con base a sus necesidades y no a caprichos o deseos.

A su vez, Johnson \& Sherraden (2007) añaden que si bien es bueno contar con información financiera, no lo es todo, pues para lograr adquirir verdaderamente una habilidad financiera es necesario ponerla en práctica y para esto se debe estar incluido financieramente y poder tener acceso al sistema financiero. 


\section{IMPORTANCIA DE LAS FINANZAS PERSONALES EN LA SALUD FINANCIERA: UNA REFLEXIÓN TEÓRICA}

Asimismo, Lusardi (2011) realizó un estudio en el cual midió la habilidad financiera, también analizó cómo se encontraban financieramente las personas al finalizar el mes, así como su planificación, elección, utilización de productos financieros y las habilidades que poseen para tomar decisiones financieras.

A continuación se describen diversos estudios que se han realizado en el campo del manejo de las finanzas personales en donde se destacan tres consecuencias que implican no contar con finanzas personales sanas:

\section{Gastar más de lo que se gana}

El endeudamiento está aumentando más rápido que la inflación, tal como lo afirman Joo y Garman (1998), ya que, para el año de 1996, más de un millón de personas se declararon en bancarrota, el número fue asombroso, pues la cifra que se obtuvo fue de $1,242,700$, un $35 \%$ más que un año anterior.

Ahora bien, para el año 1997, la cifra aumentó a 1, 350,118 consumidores en banca rota. Por lo que estudios llevados a cabo en Gran Bretaña por Atkinson \& Kempson (2004) a población entre edades de 18 y 24 años se encontró una tendencia mayor a endeudarse, lo que por consecuencia lleva a que presenten dificultades financieras debido a gastar sus ingresos en caprichos o deseos como lo son en bienes costosos, celulares o ropa de marca, con el fin de integrarse a una sociedad donde vale más la apariencia donde estos bienes se han convertido más en necesidad que un lujo (Lorgat, 2003).

Aunado a lo anterior, Anthes (2004) propone un concepto llamado mente de gratificación instantánea, la cual, la define como ese impulso que siente un individuo en gastar más en lo que quiere y no necesariamente en lo que necesita, en otras palabras, no se logra distinguir entre un deseo y una verdadera necesidad, es una mentalidad de querer todo a la voz de ya.

\section{No llevar un control de las finanzas.}

Estudios realizados por Kidwell \& Turrisi (2004) revelaron que las personas que llevaban a cabo un presupuesto tuvieron un mejor autocontrol sobre sus finanzas personales mediante la reducción de gastos innecesarios y seguir lo planeado en el presupuesto, lo cual generó una actitud favorable.

A su vez, se halló que el $46.5 \%$ de los estudiantes con un mayor grado de alfabetización financiera mantienen registros financieros detallados a diferencia de los que presentaron un menor grado de alfabetización financiera o sea un $29 \%$.

Por otro lado, otro estudio llevado a cabo por Chen y Volpe (1998) reveló que los grupos con mayor alfabetización financiera regulan sus patrones en sus gastos y decisiones al mantener un control de sus finanzas.

Asimismo, instituciones como Banamex y la UNAM en el 2014 elaboraron un estudio a jóvenes entre 15 y 29 años sobre la situación que viven en materia de cultura financiera donde se reveló que un $50 \%$ no cuenta con un control financiero adecuado en ingresos, gastos e incluso no destinan una cantidad al ahorro, entre otros.

\section{Decisiones financieras erróneas}

La incertidumbre en materia financiera que puede llevar a que un individuo pueda llegar a tomar decisiones financieras erróneas, puede evitarse al proporcionar a los colaboradores de una organización un asesoramiento financiero y así poder estos mismos llevar la gestión de sus finanzas personales de una manera óptima (Garman, Leech \& Grable, 1996).

Como se ha podido observar, entre más conoce una persona acerca de sus finanzas personales menos probabilidades tendrá de tomar decisiones financieras no acertadas, mismas que podrían 
conducir a problemas financieros tales como contratar un seguro inadecuado, no saber elegir la tarjeta de crédito ideal o seleccionar un préstamo con tasas de interés altas, gastar más de lo que se percibe monetariamente 0 tomar decisiones de inversión equívocas (Chen \& Volpe, 1998).

En este sentido, cada vez el mercado va aumentando su rango en cuanto a los productos financieros y servicios que son ofertados a inversores, usuarios o consumidores y cada vez resultan más sofisticados. De este modo, los individuos deben adoptar un mayor papel en el manejo de sus finanzas para ser capaces de comprender, por ejemplo, el riesgo-retorno que genera una inversión y así poder seleccionar la más adecuada, a esta habilidad se le conoce como educación financiera.

Por lo que, cualquier falta de conocimiento de las opciones que hay disponibles, sus características y el no tener la capacidad apropiada para elegir productos de manera óptima, podría poner en peligro las expectativas financieras de un individuo y por consiguiente su estabilidad financiera (Kumar, Barua, Jacob \& Varma, 2012).

Además, Garman et al. (1996) enlistan una serie de errores financieros y descuidos en el comportamiento de las personas que, aunque a veces son experiencias dentro de un marco normal cuando se juntan con otros eventos o catalizadores, pueden dar como resultado varios comportamientos financieros malos:

Abusar del crédito.

Ocasionalmente se quedan sin dinero.

No cuentan con un fondo de emergencia.

Ni con todas sus posesiones (activos) pueden pagar sus deudas (pasivos).

Se sienten estresados por la ausencia de dinero.

Pagan tarde sus cuentas vencidas.

Se preocupan por su seguridad laboral.

\section{CONCLUSIONES}

Como se pudo observar en este trabajo de investigación el Banco Mundial (2014) afirma que muchas personas desearían tener una mejora en sus finanzas personales, sin embargo, existe cierto grado de ignorancia en tópicos financieros, lo cual, trae como consecuencia un grado de estrés que no sólo afecta la vida financiera sino también en su hogar y trabajo (Garman et al, 1996).

Asimismo, Brown, Mount, Lamborn y Steinberg (1993) refieren que el estrés de las personas se debe a un mal uso de del crédito, malas decisiones financieras, mal manejo del dinero, un presupuesto deficiente y un sobreendeudamiento que como menciona Joo y Garman (1998), el número de personas que terminan en esta situación es cada vez mayor.

Asimismo, las causas anteriores tienen un origen. Para Chen \& Volpe (1998) y Kumar et al (2012), mencionan que esta falta de alfabetización financiera trae como consecuencia que las personas no aprovechen las herramientas que brindan las instituciones bancarias como a la hora de contratar un crédito 0 un seguro o bien, acudir a un ahorro formal, donde según datos de la CONSUSEF (2018) sólo el 40\% de la población mexicana lo realiza.

En resumen, lo anterior quiere decir que personas y su falta de conocimientos financieros generan un blanco fácil para los problemas financieras y los individuos tienden a un consumismo desmedido, no planificar metas financieras, no generar un presupuesto, no recurrir al ahorro formal 0 incluso no ahorrar para su retiro. Lo anterior, trae como resultado que las personas no puedan alcanzar un nivel de bienestar financiero (OCDE, 2013).

Sin embargo, no todo está perdido, ya que aunado a lo anterior, como afirma la OCDE (2013) la inclusión financiera ha asumido un papel muy importante pues las personas han logrado tener acceso al sistema financiero y conocer los servicios y productos que ofrece y no sólo eso, sino que junto con la educación financiera se les brindan herramientas y habilidades para que los individuos pue- 


\section{IMPORTANCIA DE LAS FINANZAS PERSONALES EN LA SALUD FINANCIERA: UNA REFLEXIÓN TEÓRICA}

dan tomar decisiones financieras correctas ante un creciente mercado financiero cada vez más sofisticado.

Según Atkinson \& Messy (2012) una persona con educación financiera tiene al menos conocimientos básicos financieros que le permiten tener un mejor control sobre sus finanzas personales, el dinero ya origina problemas, sino al contrario, logran una relación positiva con él y, por lo tanto, se manifiesta en la vida de los individuos el bienestar financiero (OCDE, 2005). Y según Kim (2000) aquellos con un bienestar financiero tienen más salud y presentan menos estrés.

No obstante, aunque el conocimiento en tópicos financieros es importante, es indispensable que el individuo cuente con habilidades financieras que le permitan desarrollar y mantener un presupuesto, un crédito o una inversión, por ejemplo (Lusardi, 2011). Zait \& Bertea (2015) definen el término habilidad financiera como la facultad de un individuo a partir de conocimientos financieros que tiene pueda llegar a tomar una decisión. Por su parte, Huston (2010) afirma que una persona con educación financiera debe contar con habilidades necesarias que le permitirán tomar decisiones asertivas en el mercado financiero y Johnson \& Sherraden (2007) mencionan que estas habilidades se pueden adquirir a través de la práctica en el sistema financiero.

Por otro lado, tal como lo menciona el Center for Financial Services Innovation (2016), un indicador para medir la salud financiera es gastar menos de lo que se gana, sin embargo, Joo \& Garman (1998) refieren que esto no es así debido las cifras de la población endeudada iban a la alza año tras año.

Por su parte, según Atkinson y Kempson (2004) esta misma tendencia se presenta entre los jóvenes entre 18 y 24 años que tienden a cumplir sus caprichos y deseos como ropa 0 celulares, asimismo, Anthes (2004) le da el nombre de gratificación instantánea. Lo anterior, quiere decir que difícilmente esta población podría alcanzar un nivel de salud financiera.

Kidwell y Turrisi (2004) revelaron que las personas que llevaban a cabo un presupuesto tuvieron un mejor autocontrol sobre sus finanzas y Chen y Volpe (1998) encontraron que los grupos con mayor alfabetización financiera regulan sus patrones en gastos y decisiones al mantener un control de sus finanzas. Lo anterior quiere decir que la alfabetización financiera genera un impacto positivo en nuestras vidas al regular de una mejor manera nuestras finanzas.

Por otro lado, la incertidumbre puede llevar a que una persona no tome las mejores decisiones y mucho menos respecto a sus finanzas personales (Garman, Leech \& Grable, 1996). Chen \& Volpe (1998) mencionan esta incertumbre puede traer consecuencias como no elegir la tarjeta de crédito idea o seleccionar un préstamos con tasas interés altas. Lo anterior, no nos garantiza aprovechar al máximo y sacarle ventaja al propio sistema financiero donde Kumar, Barua, Jacob \& Varma (201) refieren que cada vez es más sofisticado. En ese sentido, Garman et al. (1996) se presentan más errores como no contar con un fondo de emergencia o abusar del crédito.

Por último, para tener salud financiera, no sólo es importante que las personas estén incluidas financieramente para tener acceso a la amplia gama de servicios financieros que ofrece el sistema financiero sino que en conjunto con la educación financiera los individuos pueden sacar una mayor ventaja de este y adquirir mayores habilidades financiera y a su vez, tener un mayor control de sus finanzas personales a través de la educación financiera, así como tomar decisiones acertadas y bien, no gastar más de lo que ganan.

\section{REFLEXIÓN}

Como se pudo observar en el presente ensayo teórico, una población con problemas financieros trae como resultado que los individuos se endeuden con facilidad debido a que no gestionan positivamente sus finanzas personales, por ejemplo, no vivir de acuerdo a un presupuesto, no acuden al ahorro o bien, no controlan sus gastos y terminan gastando más de lo que perciben de ingresos, esto al vivir con una mentalidad de gratificación instantánea. 
En consecuencia, nuestra salud financiera se ve afectada por este tipo de comportamientos 0 actitudes. El presente ensayo teórico da pauta para investigar más acerca de la salud financiera en los individuos y como se relaciona con las variables como el comportamiento financiero, actitud financiera y a su vez el propio conocimiento financiero.

\section{REFERENCIAS BIBLIOGRÁFICAS}

Anthes, (2004). Financial illiteracy in America: a perfect storm, a perfect opportunity. Journal of Financial Service Professionals, 58(6):49-56.

Atkinson \& Messy, (2012). Measuring Financial Literacy: Results of the OECD / International Network on Financial Education (INFE) Pilot Study. Recuperado de: https://ideas.repec.org/p/oec/dafaad/15-en.html

Banamex \& UNAM, (2014). Cultura financiera de los jóvenes en México. Recuperado de: www.banamex.com/es/conoce_banamex/quienes_somos/prensa/pdf/book_brujula_digital_2014.pdf

Banco Mundial (2014). Cómo tomar control de tus finanzas personales. Recuperado de: https://blogs.worldbank.org/latinamerica/es/c-mo-tomar-el-control-de-tus-finanzas-personales

Banco Mundial (2018). La inclusión financiera es un factor clave para reducir la pobreza e impulsar la prosperidad. Recuperado de: https://www.bancomundial.org/es/topic/financialinclusion/overview

Billimoria, Penner y Knoote, (2013). Uso de los servicios financieros. Estudio empírico de estudiantes universitarios. International Journal of Delelopmental and Educational Psicology. Disponible en: http://www.redalyc.org/articulo.oa?id=349853220056

Borghino, (2012). El arte de hacer dinero. México. Penguin Random House Grupo Editorial México. Primera edición.

Brown, Mount, Lamborn y Steinberg, (1993). Parenting practices and peer group affiliation in adolescence. Child Development, 64, 467-482.

Center for Financial Services Innovation, (2016). Eight ways to Measure Finantial Heatlh. Recuperado de: www.bbvaedufin.com/publicacion/ocho-indicadores-para-medir-la-salud-financiera/

Chen y Volpe, (1998). An analysis of personal financial literacy among college students. Financial Services Review, 7(2), 107-128.

Chinen \& Endo, (2012). Effects of attitude and background on personal financial ability: A student survey in the United States. International Journal of Management, 29(1), 33.

CNBV, (2009). Primer Reporte de Inclusión Financiera, México.

CONDUSEF, (2018). ¿Cómo ahorramos los mexicanos? Recuperado de: www.condusef.gob.mx/Revista/index.php/ahorro/ahorro/168-como-ahorramos-los-mexicanos

Danes \& Hira, (1987). Money Management Knowledge of College Students. Journal of Student Financial Aid, 17(1), 4-16.

FUNDEF, (2017). ¿Cómo se da la inclusión financiera? Análisis sobre la dinámica de acceso al crédito formal en México. Recuperado de: http://fundef.org.mx/sites/default/files/fundeforgmx/ paginas/archivos/\%3Cem\%3EEditar\%20P\%C3\%A1gina\%20b\%C3\%A1sica\%3C/em\%3E \%20D ocumentos $\% 20 \mathrm{de} \% 20 \mathrm{Coyuntura} \% 20$ Estructural/documentoinclusionfinancierafundef.pdf

Garman, Leech y Grable, (1996). The negative impact of employee poor personal financial behaviours on employers. Financial Counseling and Planning, 7:157-168.

Garman y Forgue, (1997). Personal finance (5th ed.). Boston: Houghton Mifflin Company. Recuperado de: http://weraby.org/files/personal_finance_5th_edition_kapoor.pdf

Huston, (2010). Measuring Financial Literacy. The journal of consumer affairs, 44(2), 296-316.

Joo y Garman, (1998). The Pottencial Effects of Workplace Financial Education. Personal Finances 
and Worker productivity. Vol. II No. 1 Pp 1-2. Available at: http://citeseerx.ist.psu.edu/viewdoc/download?doi=10.1.1.564.8153\&rep=rep1\&type=pdf

Joo, (1998). Personal financial wellness and worker job productivity. Unpublished doctoral dissertation, Virginia Polytechnic Institute y State University, Blacksburg.

Johnson \& Sherraden, (2007). From financial literacy to financial capability among youth. Journal of Sociology and Social Welfare, 34(3), 119-145.

Kidwell \& Turrisi (2004). An examination of college student money management tendencies. Journal of Economic Psychology, 25(5):601-616.

$\mathrm{Kim},(2000)$. The effects of workplace financial education on personal finances and work outcomes. Unpublished doctoral dissertation, Virginia Polytechnic Institute y State University, Blacksburg.

Kumar; Barua; Jacob, Varma, (2012). A survey of financial literacy among students, Young employees and the retired in India. Disponible en: https://faculty.iima.ac.in/ iffm/literacy/youngemployessandretired2012.pdf

León, (2006). Aptitud financiera y bienestar financiero de los empleados de la Universidad de Montemorelos. Tesis de maestría. Universidad de Montemorelos. Facultad de Ciencias Administrativas. Nuevo León, México.

Lorgat, (2003). Enabling worker finances: the effects of rising costs of living and debt on worker finances. South African Sociological Association. Durban.

Munohsamy, (2005). Personal Financial Management. University of Brunei. Disponible en: https://www.researchgate.net/publication/279198054

Lusardi y Mitchell, (2007). Financial education and Inclusion in Latin America and the Caribbean. Programs of Central Banks and Financial Superintendencies. Disponible en: http://www.cemla.org/PDF/otros/2014-10-Financial-Education-Inclusion-LAC.pdf

Lusardi, (2009). The Importance of Financial Literacy. NBER Reporter, 2, 13-16.

Lusardi y Mitchell (2011). Financial Literacy Around the World: An Overview, Cambridge, MA, National Bureau of Economic Research.

Lusardi, (2011). Americans' financial capability. National Bureau of Economic Research Working Paper No. w17103. DOI: 10.3386/w17103

OCDE (Organización para la Cooperación y Desarrollo Económico), (2005). Recommendation on Principles and Good Practices for Financial Education and Awareness.

OCDE, Banco de la República, Caf, y Fogafin (2013), Financial Education in Latin America and the Caribbean: Rationale and Way Forward, International Network on Financial Education, Prague.

OCDE, (2013). Advancing National Strategies for Financial Education. Russia's G20 Presidency and the OCDE.

O'Neill, Sorhaindo, Xiao y Garman (2005). Financial Distressed Consumers: Their Financial Practices, Financial Well-being, and Health. Association for Financial Counseling and Planning Education, 16 (1). Recuperado en: http://papers. Ssrn.com/sol3/papers.cfm? abstract_id=2255121.

Oton y Torrento, (2017). Employees' Financial Literacy, Behavior, Stress and Wellness. Journal of Human Resource Management, 5(5), 78-89. doi: 10.11648/j.jhrm.20170505.12

Zait y Bertea, (2015). Financial Literacy-Conceptual Definition and Proposed Approach for a Measurement Instrument. The Journal of Accounting and Management, 4(3).

Zakaria, Jaafar y Marican (2012), "Financial behavior and financial position: a structural equation modelling approach", en Middle-East Journal of Scientific Research, vol. 12, núm. 10, pp. 13961402. 